\title{
CDISC SDTM Tumor and Lesion Identification Result Terminology
}

National Cancer Institute

\section{Source}

National Cancer Institute. CDISC SDTM Tumor and Lesion Identification Result

Terminology. NCI Thesaurus. Code C96780.

Terminology associated with the tumor identification result codelist of the Clinical Data Interchange Standards Consortium (CDISC) Study Data T abulation Model (SDT M). 